\title{
Evaluación del nivel de competencia comunicativa escritora en estudiantes universitarios
}

\section{Evaluation of the level of communicative writing competence in university}

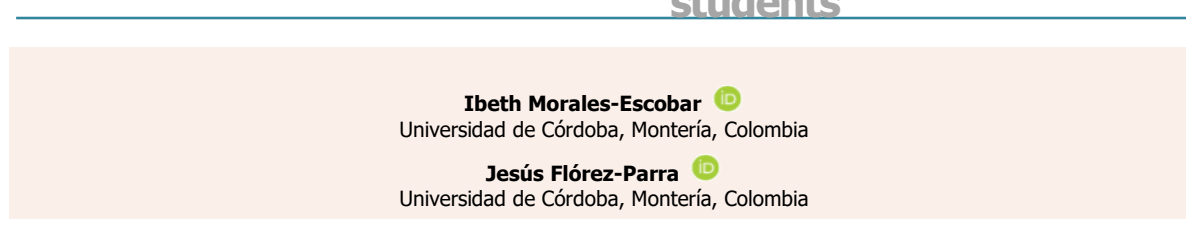

\section{Resumen}

Objetivo: Evaluar el nivel de la competencia comunicativa escritora en estudiantes universitarios. Método: Enfoque cuantitativo con método no experimental, aplicando una rejilla para evaluar las variables cohesión, coherencia, adecuación y superestructura en un ensayo escrito por estudiantes que cursaron dos niveles de Competencias Comunicativas. Resultados: El 54\% de los estudiantes hizo buen uso de los procedimientos anafóricos léxicos y gramaticales; el $52 \%$ hizo uso regular de los conectores lógicos; el 39\% usó bien los signos de puntuación; el $51 \%$ escribió textos con nivel regular de coherencia; el $40 \%$ estructuró textos en un nivel regular de acuerdo con la tipología y el $47 \%$ presentó un nivel regular en la adecuación textual. Discusión y Conclusiones: Los estudiantes presentan un nivel bueno en el uso de mecanismos de cohesión por lo que estructuran textos en un nivel microestructural. Pero, las formas de hacer coherente un escrito, estructurarlo y hacerlo adecuado constituyen una dificultad para ellos. Se necesita mejorar las habilidades para hacer del texto un todo coherente y adecuado al contexto, por tanto, el programa debe tener en cuenta estas consideraciones.

Palabras clave: Competencia Comunicativa, Escritura, Evaluación, Enseñanza superior.

\begin{abstract}
Objective: Evaluate the level of communicative writing competence in university students. Method: Quantitative approach with a non-experimental method, applying a grid to evaluate the variables cohesion, coherence, adequacy and superstructure in an essay written by students who studied two levels of Communicative Competences. Results: $54 \%$ of the students made good use of lexical and grammatical anaphoric procedures; $52 \%$ made regular use of logical connectors; $39 \%$ made good use of punctuation marks; $51 \%$ wrote texts with a regular level of coherence; $40 \%$ structured texts at a regular level according to the typology and $47 \%$ presented a regular level in textual adequacy; and $47 \%$ presented a regular level of coherence. Discussion y Conclusion: The students present a good level in the use of cohesion mechanisms, so they structure texts at a microstructural level. However, the ways to make a text coherent, to structure it and make it adequate constitute a difficulty for these students. It is necessary to improve the skills to make the text a coherent whole and adequate to the context, therefore, the program should take into account these considerations.
\end{abstract}

Keywords: Communication Competence, Writing, Assessment, Higher level education

Open Access:

ISSN: $0124-2121$ E-ISSN: $2665-2420$

Editor: Dhayana Fernández Matos

TIPOLOGÍA DE ARTÍCULO Copyright ${ }^{\circ}$ By Educación y Humanismo 


\section{Introducción}

En los últimos años se han desarrollado investigaciones relacionadas con los procesos de comprensión y producción textual en estudiantes universitarios, tanto en Colombia como en Latinoamérica y España. Estas se han centrado en distintos propósitos: algunas en la descripción de las prácticas de lectura y escritura que se desarrollan en las asignaturas disciplinares de las distintas carreras (González y Vega, 2010; Colciencias, 2013; Eudaye, Carvajal, Macías, Páez y Cañedo, 2019). Estas indican que normalmente se llevan a cabo prácticas que conducen a resolver necesidades académicas inmediatas y no permanentes, como estudiar para un examen, hacer una exposición, responder a cuestionamientos de los docentes, entre otras.

Otro tipo de investigaciones ha indagado las percepciones, actitudes y habilidades que tienen los universitarios a la hora de leer y escribir, a través de pruebas de comprensión y producción textual (Rivera, Cornejo y Roco, 2008; Andrade, 2009; Ramírez, Tánori, García y Urías, 2017; Flores, 2018; Rojas y Rodríguez, 2018). Los resultados demuestran falencias en los estudiantes, ya que no logran alcanzar los niveles requeridos de lectura y escritura para su quehacer académico en la universidad.

Por último, otros estudios se han propuesto diseñar programas y estrategias de intervención que sirvan de métodos de enseñanza para las habilidades de lectura y escritura en estudiantes de Educación Superior (Ávila, González y Peñaloza, 2013; Díaz y Morales, 2013; Moyano, 2016, 2018; Londoño y Ospina, 2018; Suárez, 2019).

Sin embargo, son pocas las investigaciones publicadas en revistas científicas indexadas que tengan por objetivo evaluar el nivel de desarrollo de las competencias comunicativas de los estudiantes luego de cursar una asignatura de competencias comunicativas (o de comprensión y producción de textos). Entre estas, Molina y López (2020) identificaron las teorías, prácticas y procesos de gestión de los centros y programas de escritura y concluyeron que "la evaluación de los programas de escritura parece ser una actividad que la mayoría de los programas aún no lleva a cabo" (Molina y López, 2020, p. 110).

En este marco, surge la necesidad de evaluar el nivel de desarrollo de la competencia comunicativa escritora de los estudiantes de la Licenciatura en Literatura y Lengua Castellana de la Universidad de Córdoba luego de cursar la asignatura Competencias Comunicativas.

Esta investigación se fundamenta y analiza los resultados desde la perspectiva de teorías y conceptos propios de la lingüística cognitiva, textual y discursiva (Hymes, 1972; Flower y Hayes, 1996; Cassany, Luna y Sanz, 2003; Van Dijk, 1992, 1996; Sánchez, 2000; Serafini, 1992 y García, 2015). 
Dado el carácter evaluativo de la pesquisa que aquí se plantea, se ha seguido una metodología de enfoque cuantitativo con método ex post facto. De modo que, al llevarse a cabo la evaluación un semestre después de haber sido cursada la asignatura, no se realizó ningún tipo de modificaciones sobre la variable en estudio.

\section{Competencia comunicativa}

El término competencia hace referencia a la "pericia, aptitud o idoneidad para hacer algo o intervenir en un asunto determinado" (RAE, 2020), lo que, aterrizado al campo educativo, se convierte en el punto de partida de todo el proceso de enseñanza y aprendizaje, pues es necesario responder, en primera instancia, qué capacidades deben tener los estudiantes para participar activamente en la sociedad y, por tanto, cómo ayudar al desarrollo de estas desde el currículo. En este sentido, dichas capacidades tienen que favorecer el desarrollo integral de los sujetos al coadyuvarles en la evaluación y elección de las alternativas más eficaces para circunstancias específicas de su vida (Fernández, 2019). De ahí surge la necesidad de desarrollar adecuadamente una competencia comunicativa que permita saber qué comunicar y cómo hacerlo para el éxito personal y social.

Para Hymes (1972), la competencia comunicativa se entiende no solo como el conocimiento estructural de la lengua, sino también como la habilidad para el uso de la misma que posee el hablante en contextos determinados. Esto indica que para comunicarnos se deben tener en cuenta factores como: a quién hablar, cuándo hacerlo, qué decir y en qué situación concreta. Es decir, la comunicación es un proceso en el que, aparte de las reglas gramaticales, es necesario tener en cuenta los factores socioculturales que entran en juego cuando esta se produce.

Para el caso de la lectura y la escritura, este concepto instaura las directrices que regulan dichos procesos en los estudiantes, ya que todo texto se circunscribe en un contexto determinado $y$, en la configuración de este, se deben tomar en cuenta los factores sociales para su adecuada interpretación.

Ahora bien, la competencia comunicativa engloba otras competencias (Pérez, 2006) como: competencia lingüística, entendida como el conocimiento formal de la lengua; competencia textual, referida a la comprensión y producción de diferentes tipos de textos; competencia pragmática, enfocada en el uso del lenguaje según el contexto; competencia argumentativa, centrada en aportar las razones y pruebas para defender opiniones, ideas, concepciones o comportamientos; y, la competencia enciclopédica, concerniente a los saberes de distintas disciplinas que usamos cuando nos comunicamos.

Cabe aclarar, en este punto, que las asignaturas a través de las cuales se desarrollan y fortalecen las competencias de lectura y escritura en las universidades colombianas reciben diferentes denominaciones, entre ellas: comprensión y producción de textos, taller de lengua, escritura argumentativa, lectura crítica y competencias comunicativas. Por esta 
razón, en esta investigación, cuando se hable de competencia escritora y de los procesos de producción textual, se hace referencia también a la competencia comunicativa, en general, aun cuando no se esté evaluando el componente de lectura y oralidad.

\section{Competencia escritora}

Al igual que la lectura, la escritura en la Educación Superior es una actividad compleja, la cual requiere el uso de habilidades cognitivas que permitan codificar información de manera coherente, de acuerdo con las exigencias de la situación comunicativa donde se inserte la producción de un texto. La competencia escritora se hace patente en la producción textual, que según el MEN (2006): "hace referencia al proceso por medio del cual el individuo genera significado, ya sea con el fin de expresar su mundo interior, transmitir información o interactuar con los otros"(p. 20).

El acto de escribir no se limita al conocimiento de la gramática o los signos gráficos, como se entendía de manera tradicional. Al respecto, el MEN (1998), sostiene que:

No se trata solamente de una codificación de significados a través de reglas lingüísticas. Se trata de un proceso que a la vez es social e individual en el que se configura un mundo y se ponen en juego saberes, competencias, intereses, y que a la vez está determinado por un contexto socio-cultural y pragmático que determina el acto de escribir: escribir es producir el mundo (...) Pero es claro que el hecho de comprender el acto de escribir como producción de la significación y del sentido no excluye el componente técnico, lingüístico y comunicativo del lenguaje; las competencias asociadas al lenguaje encuentran su lugar en la producción del sentido (p. 27)

En este sentido, la competencia escritora se puede entender como la habilidad para producir diferentes tipos de texto, en los que se generan significados con un propósito determinado. Sin embargo, dicha generación de sentidos solo se alcanza cuando se conoce la forma de construir el texto, de transmitir las ideas y el contexto en el que dicho texto se produce, esto es, la situación de comunicación. De acuerdo con esta conceptualización, a la escritura le subyace un conocimiento de la lengua de tipo morfosintáctico, semántico y pragmático.

Es importante tener en cuenta que la escritura, en tanto que manifestación de la comunicación humana, es un proceso y no un producto, como se consideraba en algunos modelos de producción textual tradicionales. La escritura como producto se entiende como un conjunto de etapas lineales que se siguen para producir un texto; en cambio, la escritura como proceso se define en cuanto a las operaciones mentales que se siguen para producir dicho texto, en un ciclo de permanente revisión. Entre los modelos procesuales, el más conocido es el de Flower y Hayes (1996), en el que se sostiene que la escritura se lleva a 
cabo en un proceso que incluye varias etapas, pero no de manera lineal, sino que, durante la composición del texto se puede reconstruir lo que ya se ha escrito.

En este modelo se proponen tres etapas: planificación, en la que "los escritores forjan una representación interna del conocimiento que utilizarán durante la escritura" (Flower y Hayes, 1996, p. 7), es decir, concebir las ideas y determinar cuáles son las más adecuadas, luego organizarlas y estructurarlas para que tengan sentido, después fijar objetivos para dar cuenta del cómo y para qué va a escribir; traducción, en esta se convierten en lenguaje visible las ideas generadas en la planificación, teniendo en cuenta las exigencias especiales de la lengua escrita (gramática, léxico, etc.); la revisión, en la cual se lee lo que se ha escrito (traducido), bien sea para hacer traducciones posteriores, o con el objetivo de evaluar sistemáticamente el texto para verificar su coherencia; $y$, finalmente, el control, que se realiza durante toda la elaboración del texto para dirigir el proceso y su progreso, así como determinar cuándo se pasa de una etapa a otra (Flower y Hayes, 1996).

De este modo, se podría definir la escritura como un proceso por medio del cual el escritor elabora una obra final a la que llamamos texto, teniendo la posibilidad de "ir y venir" a lo largo del proceso de composición. Así pues, la competencia escritora incluye la habilidad para redactar adecuadamente, con base en los procesos y subprocesos que orientan la producción y generación de sentido y significado. Algunas investigaciones han demostrado la eficacia de este modelo para mejorar el nivel de desempeño de los estudiantes en la escritura académica, así como para fomentar las habilidades de comprensión y producción de textos (Briceño, 2014; Medina y Leal, 2019; Moreno, 2020).

\section{Propiedades textuales}

La competencia escritora se evidencia, como ya se ha mencionado, cuando un estudiante es capaz de construir un texto de manera coherente y según las exigencias del contexto de producción. Esta construcción se logra si el estudiante tiene un amplio conocimiento de las propiedades textuales, que, según Cassany, Luna y Sanz (2003, p. 315), son "todos los requisitos que ha de cumplir cualquier manifestación verbal para poder considerarse un texto y, por lo tanto, para poder vehicular el mensaje en un proceso de comunicación". Las propiedades textuales que se estudian son tres: cohesión, coherencia y adecuación; y, aunque, la superestructura hace parte de la coherencia, se ha decidido tomarla aparte por la peculiaridad de su función en la comunicación escrita (la de caracterizar el tipo de texto).

La cohesión "se refiere a la técnica y a los procedimientos de construcción del texto, y consiste en que las proposiciones, las oraciones y los párrafos estén ensamblados y se den sentido entre sí utilizando diversos procedimientos léxicos, semánticos y gramaticales" (Sánchez, 2000, p. 240). Así pues, un texto queda bien estructurado y es comprensible cuando todas sus partes están bien cohesionadas.

Al respecto, Cassany, Luna y Sanz (2003), proponen que: 
Las oraciones que conforman un discurso no son unidades aisladas e inconexas, puestas una al lado de otra, sino que están vinculadas o relacionadas con medios gramaticales diversos (puntuación, conjunciones, artículos, pronombres, sinónimos, entonación, etc.), de manera que conforman entre sí una imbricada red de conexiones lingüísticas, la cual hace posible la codificación y descodificación del texto (p. 323)

Las principales formas de cohesión son: la anáfora, los conectores lógicos y los signos de puntuación (Serafini, 2011; Sánchez, 2000; Cassany et. al., 2003; García 2015). La anáfora consiste en la repetición de un mismo elemento en oraciones sucesivas mediante el uso de referencias gramaticales como los pronombres, adverbios y artículos. Entre los principales mecanismos de referencia anafórica se encuentran: la sustitución léxica, mediante la sinonimia y la generalización; la elipsis o supresión de un elemento conocido que aparece muy cerca del original en el texto y que el receptor puede reconstruir (sujetos, complementos, etc.); y, los determinantes, es decir artículos (un, la, el), adjetivos demostrativos (este, aquel) y posesivos (mi, tu, su), etc., que establecen varios tipos de relaciones entre las palabras y las referencias: desconocido/conocido, emisor/receptor, cercano/ lejano, etc.

Los conectores lógicos establecen diversos y numerosos tipos de relaciones (coordinación/subordinación, oposición significativa, ordenación lógica, temporal, causaefecto, etc.) entre los enunciados o partes del texto, que se expresan mediante varios tipos de enlace (Cassany et. al., 2003) los cuales permiten dotar de unidad semántica al texto. De acuerdo con García (2015):

Los conectores o formas gramaticales no aportan un contenido específico, pero sí expresan una referencia lógica. Se utilizan como indicadores funcionales para organizar el texto, para enfatizar puntos clave o decisivos, para debatir los contenidos, para interpretarlos como instrucciones, etc. También sirven para explicar causas, argumentar con razones y oponerlas a las que sustentan la información ( $p$. 252)

Los signos de puntuación "señalan los diversos apartados del escrito y conforman un auténtico esqueleto jerárquico del texto" (Cassany et. al., 2003, p. 326). En este sentido, su función "consiste en subdividir el texto según su estructura semántica y sintáctica, de modo que facilite la comprensión e interpretación de lo escrito" (Serafini, 1994, p. 239). En definitiva, los signos de puntuación dotan al texto de una organización sintáctico-semántica que orienta al lector y le permite la comprensión eficaz de aquel.

Por su parte, la coherencia atañe al significado del texto y para que este sea coherente "... debe mantener un equilibrio entre su fondo o contenido, su forma o manera de transmitir la información y su estructura lógica" (Sánchez, 2000, p. 239). En otras palabras, la 
coherencia hace referencia a la forma en la que se procesa la información para darle sentido global al texto. Al respecto, Cassany et. al. (2003, p. 319) plantean que:

La coherencia establece cuál es la información pertinente que se ha de comunicar y cómo se ha de hacer (en qué orden, con qué grado de precisión o detalle, con qué estructura, etc.). Por ejemplo, las redacciones o las exposiciones de los alumnos que son desorganizadas, que repiten ideas y las mezclan, y que no dicen las cosas de forma ordenada, aquellas que no tienen ningún esquema -diríamos-, son textos incoherentes.

Un texto no será coherente si las ideas expresadas en él no están bien conectadas, esto es, no están cohesionadas. Es imprescindible, entonces, conocer los mecanismos de cohesión que permiten darle un sentido global al texto que se produce. Según Cassany et., al. (2003), los aspectos más importantes que incluye la coherencia textual son la cantidad de la información, la calidad de esta y su estructuración.

La cantidad de la información se centra en aspectos como: ¿cuál es la información pertinente o relevante para cada tipo de comunicación?, ¿se dicen todos los datos que se han de decir, no hay ni exceso de información (repetición, redundancia, paja, datos irrelevantes para el propósito comunicativo, etc.) ni defecto (lagunas en el significado, exceso de presuposiciones o de datos implícitos que el receptor no domina)?

La calidad de la información responde a los siguientes interrogantes: ¿es buena la información del texto?, ¿las ideas son claras y comprensibles, se exponen de forma completa, progresiva y ordenada, con los ejemplos apropiados, la terminología específica y las formulaciones precisas? o, por el contrario, ¿se detectan ideas oscuras, falta de concreción, enunciados demasiado genéricos y teóricos o excesivamente anecdóticos?

La estructuración de la información da respuesta a preguntas como: ¿cómo se organiza la información del texto?, ¿los datos se estructuran lógicamente según un orden determinado (cronológico, espacial, etc.)?, ¿cada idea se desarrolla en un párrafo o en una unidad independiente?, ¿hay una introducción inicial y un resumen final?, ¿la información nueva se administra de forma progresiva?; si, por ejemplo, se trata de un ensayo ¿el texto contiene los apartados típicos de este tipo de texto, es decir: una tesis, un cuerpo de argumentos y una conclusión?

Se puede decir, entonces, que en la medida en que un escritor encuentre el equilibrio entre estos tres aspectos (calidad, cantidad y estructuración) de la información que va a procesar y plasmar en su texto, logrará que este sea coherente y, por tanto, comprensible para sus lectores.

Como última propiedad textual, la adecuación demanda que la forma de comunicación se adapte al tema tratado, a las intención comunicativa y al nivel de formalidad que el autor desea dar al texto (Sánchez, 2000). Al respecto, Cassany et. al. (2003, p. 317) proponen 
que la adecuación significa "saber escoger de entre todas las soluciones lingüísticas que te da la lengua, la más apropiada para cada situación de comunicación".

Por lo tanto, consideran que, para saber si un texto consigue la adecuación necesaria, hay que analizar algunos aspectos. En primer lugar, fijarse si este consigue realmente el propósito comunicativo por el cual ha sido producido (por ejemplo: informar de un hecho, exponer una opinión, solicitar alguna información, etc.). De igual forma, comprobar si se mantiene el mismo nivel de formalidad, sea alto o bajo, durante todo el texto. Finalmente, verificar si se conserva el mismo grado de especificidad, sea también alto o bajo, durante todo el texto. Si el texto trata de un tema especializado, es lógico que se utilice la terminología específica del campo y que se eviten las expresiones coloquiales y las palabras generales porque pierde precisión.

Así, la adecuación se equipara con el conocimiento pragmático de la lengua, pues el funcionamiento y uso del lenguaje para la configuración del texto van a depender de una situación de comunicación específica. Esta situación se refiere al contexto de la actividad, es decir, que la actividad del escritor debe regirse por las normas de una comunidad discursiva (Martínez, Álvarez, Hernández, Zapata y Castillo, 2004).

En definitiva, la buena competencia escritora se puede apreciar cuando se cumplen los requisitos anteriormente dados, esto es, que todo texto obedezca a unas exigencias lingüísticas y comunicativas de cohesión, coherencia y adecuación que permitan construir significados para establecer un intercambio social con lectores en un contexto determinado.

\section{Método}

Esta investigación se inserta en la perspectiva cuantitativa, que está directamente basada en el paradigma explicativo y utiliza información cuantificable para describir o tratar de explicar los fenómenos que estudia (Briones, 1996). Se sitúa en el método no experimental, en el cual no se tiene control alguno sobre la variable independiente, pues, cuando el investigador ha realizado el estudio, ésta ya se ha presentado (Briones, 1996). En este caso, se estudia el nivel de la competencia escritora de los estudiantes, que constituye la variable dependiente, después de haber cursado el programa de competencias comunicativas, el cual representa la variable independiente.

Para la recolección de la información se empleó una rejilla de evaluación de textos académicos, diseñada con base en las propiedades textuales de cohesión, coherencia, adecuación y superestructura. Estas propiedades se concretaron en una serie de indicadores que dan cuenta de la adecuada construcción de un texto académico, los cuales se calificaron así: M (Malo), R (Regular), B (Bueno) y E (Excelente), como se observa en la tabla 1. 
Tabla 1.

Rejilla de evaluación de textos académicos

\begin{tabular}{|c|c|c|c|c|}
\hline \multirow{2}{*}{$\begin{array}{l}\text { PROPIEDADES } \\
\text { TEXTUALES }\end{array}$} & \multirow{2}{*}{ INDICADORES } & \multicolumn{3}{|c|}{ CALIFICACIÓN } \\
\hline & & $\mathbf{R}$ & B & $\mathbf{E}$ \\
\hline Cohesión & $\begin{array}{l}\text { Hace uso de la sustitución léxica por medio de sinónimos. } \\
\text { Pronominaliza para evitar la redundancia. } \\
\text { Suprime sustantivos y verbos cuando su presencia se } \\
\text { sobreentiende en el texto. } \\
\text { Usa los conectores lógicos que corresponden a la relación } \\
\text { semántica entre las ideas. } \\
\text { Usa el punto para separar ideas con sentido completo y los } \\
\text { párrafos de un texto. } \\
\text { Usa los distintos tipos de coma (enumerativa, vocativa, } \\
\text { hiperbática, incidental, elíptica) para delimitar unidades } \\
\text { lingüísticas inferiores al enunciado. } \\
\text { Usa el punto y coma en situaciones de yuxtaposición y } \\
\text { enumeración compleja. } \\
\text { Hace uso adecuado de otros signos de puntuación y la } \\
\text { ortografía en general. }\end{array}$ & & & \\
\hline Coherencia & $\begin{array}{l}\text { Las ideas son claras y comprensibles. } \\
\text { Hay relación semántica adecuada entre las proposiciones de } \\
\text { la secuencia discursiva. } \\
\text { Las ideas se exponen de forma completa, progresiva y } \\
\text { ordenada. } \\
\text { Cada idea se desarrolla en un párrafo o unidad } \\
\text { independiente. } \\
\text { La información que expone en las partes del texto es } \\
\text { pertinente para el tema global. } \\
\text { Introduce citas en el texto y las parafrasea o explica de } \\
\text { manera precisa. }\end{array}$ & & & \\
\hline Superestructura & $\begin{array}{l}\text { Jerarquiza y organiza las ideas del texto de acuerdo con la } \\
\text { tipología del mismo. } \\
\text { Cada parte de la superestructura cumple con su función. }\end{array}$ & & & \\
\hline Adecuación & $\begin{array}{l}\text { El texto consigue el propósito comunicativo para el cual ha } \\
\text { sido producido. } \\
\text { Se mantiene un mismo nivel de lenguaje durante todo el } \\
\text { texto. } \\
\text { El texto responde a la situación de enunciación en la que } \\
\text { fue producido. }\end{array}$ & & & \\
\hline
\end{tabular}

Fuente: elaboración propia (2021)

Este instrumento permitió evaluar la competencia escritora de los estudiantes, mediante la valoración de un ensayo producido por ellos en una asignatura del plan de estudio de la carrera. Se escogió el ensayo porque es una de las principales tipologías textuales que con mayor frecuencia producen los estudiantes de la Licenciatura en Literatura y Lengua Castellana.

La población objeto de estudio fueron los estudiantes matriculados en tercer semestre de la Licenciatura en Literatura y Lengua Castellana de la Universidad de Córdoba. Para la selección de la muestra se llevó a cabo un muestreo intencional, escogiendo un grupo de 50 estudiantes de la asignatura Proyecto de Investigación, la cual es un curso obligatorio de la carrera, tiene como prerrequisito las asignaturas de competencias comunicativas y requiere un nivel adecuado de la competencia escritora para la redacción de un proyecto de investigación. 
El análisis de la información se realizó mediante la estadística descriptiva, que permitió organizar, describir y presentar el conjunto de datos relacionados con el nivel de escritura de los estudiantes a través de tablas de frecuencia.

\section{Resultados}

Se exponen los resultados del análisis textual a un ensayo escrito por los estudiantes en el que se evaluó su competencia escritora con base en su conocimiento y aplicación de las propiedades textuales de cohesión, coherencia, adecuación y superestructura. Para ello, se presenta el análisis de los indicadores en cuatro apartados, cada uno correspondiente a las propiedades mencionadas.

\section{Cohesión}

Teniendo en cuenta que hay tres mecanismos principales de cohesión textual, se expone, a continuación, el análisis del uso de cada uno de ellos: las referencias léxicas y gramaticales, los conectores lógicos y los signos de puntuación.

\section{Tabla 2}

Frecuencia de uso de referencia léxica y gramatical

\begin{tabular}{|c|c|c|c|c|c|}
\hline \multirow{2}{*}{ INDICADOR } & \multicolumn{4}{|c|}{ CALIFICACIÓN } & \multirow{2}{*}{ TOTAL } \\
\hline & $\mathbf{M}$ & $\mathbf{R}$ & B & $\mathbf{E}$ & \\
\hline Hace uso de la sustitución léxica por medio de sinónimos. & 2 & 22 & 24 & 2 & 50 \\
\hline Pronominaliza para evitar la redundancia. & 4 & 18 & 26 & 2 & 50 \\
\hline $\begin{array}{l}\text { Suprime sustantivos y verbos cuando su presencia se } \\
\text { sobreentiende en el texto. }\end{array}$ & 2 & 16 & 30 & 2 & 50 \\
\hline Porcentaje & $5 \%$ & $37 \%$ & $54 \%$ & $4 \%$ & $100 \%$ \\
\hline
\end{tabular}

Fuente: elaboración propia (2021)

Como se puede apreciar en la tabla 2, el 54\% de los estudiantes de la muestra hacen buen uso de los procedimientos anafóricos léxicos y gramaticales, el 37\% lo hace de manera regular, el $4 \%$ de manera excelente y el $5 \%$ lo hacen mal. Entonces, se puede aseverar que, al momento de escribir, la mayoría de los estudiantes utilizan bien los mecanismos de referenciación léxica y gramatical, como la pronominalización, la sinonimización y la elisión de elementos cuya presencia se sobreentiende en el texto.

Es de vital importancia que los estudiantes conozcan estos mecanismos porque así evitan repeticiones innecesarias y redundancias que puedan tornar confuso el texto. Si no dispusieran de ellos y no los usaran adecuadamente, las frases serían incompletas y se dificultaría la comprensión para los lectores (Cassany et. al., 2003). 


\section{Uso de los conectores lógicos}

Tabla 3

Frecuencia de uso de conectores lógicos

\begin{tabular}{cccccc}
\hline \multicolumn{1}{c}{ INDICADOR } & \multicolumn{3}{c}{ CALIFICACIÓN } & \multicolumn{2}{c}{ TOTAL } \\
\cline { 2 - 4 } & $\mathbf{M}$ & $\mathbf{R}$ & $\mathbf{B}$ & $\mathbf{E}$ \\
\hline $\begin{array}{l}\text { Usa los conectores lógicos que corresponden a la relación } \\
\text { semántica entre las ideas. }\end{array}$ & 10 & 26 & 12 & 2 & 50 \\
\hline Porcentaje & $20 \%$ & $52 \%$ & $24 \%$ & $4 \%$ & $100 \%$ \\
\hline
\end{tabular}

Fuente: elaboración propia (2021)

De acuerdo con la tabla anterior, el $52 \%$ de los estudiantes hacen un uso regular de los conectores lógicos, el $24 \%$ lo hace bien, el $20 \%$ mal y solo el $4 \%$ lo hace de manera excelente. Lo anterior evidencia que la mayor parte de los estudiantes presentan un nivel regular en cuanto al uso de los conectores lógicos, para enlazar las ideas dentro del párrafo y entre los párrafos, pues, o bien no los utilizan, o los que utilizan no siempre son los más apropiados para establecer las debidas relaciones lógicas (causa-efecto, contraste, adición, orden, etc.).

Al respecto, se observó que, en algunos casos, por ejemplo, utilizan conectores como ya que, así pues, por otro lado, etc.; sin embargo, estos no establecen la relación lógica correspondiente, lo que demuestra una falta de destreza para identificar las relaciones semánticas entre las partes del texto, pues cuando se trata de usar conectores, no lo hacen en los contextos adecuados.

Nótese que el uso regular $(R)$ de los conectores lógicos demuestra las dificultades generales para organizar el texto y conectar las proposiciones de la secuencia discursiva, ocasionando que se torne confusa la idea general del párrafo y/o la unidad de sentido de todo el texto. En un ensayo, como texto argumentativo, es necesaria la presencia de estos enlaces, pues "sirven para explicar causas, argumentar con razones y oponerlas a las que sustentan la información" (García, 2015. p. 252).

Tabla 4.

Frecuencia de uso de signos de puntuación

\begin{tabular}{|c|c|c|c|c|c|}
\hline \multirow{2}{*}{ INDICADOR } & \multicolumn{4}{|c|}{ CALIFICACIÓN } & \multirow{2}{*}{ TOTAL } \\
\hline & $\mathbf{M}$ & $\mathbf{R}$ & B & $\mathbf{E}$ & \\
\hline $\begin{array}{l}\text { Usa el punto para separar ideas con sentido completo y los } \\
\text { párrafos de un texto. }\end{array}$ & 6 & 18 & 24 & 2 & 50 \\
\hline $\begin{array}{l}\text { Usa los distintos tipos de coma (enumerativa, vocativa, } \\
\text { hiperbática, incidental, elíptica) para delimitar unidades } \\
\text { lingüísticas inferiores al enunciado. }\end{array}$ & 4 & 12 & 31 & 3 & 50 \\
\hline $\begin{array}{l}\text { Usa el punto y coma en situaciones de yuxtaposición y } \\
\text { enumeración compleja. }\end{array}$ & 44 & 2 & 4 & 0 & 50 \\
\hline $\begin{array}{l}\text { Hace uso adecuado de otros signos de puntuación y la } \\
\text { ortografía en general. }\end{array}$ & 4 & 26 & 20 & 0 & 50 \\
\hline Porcentaje & $29 \%$ & $29 \%$ & $39 \%$ & $3 \%$ & $100 \%$ \\
\hline
\end{tabular}

Fuente: elaboración propia (2021) 
En relación con el uso de los signos de puntuación, el 39\% hace buen uso de ellos, un $29 \%$ lo hace mal, otro $29 \%$ lo hace regular y solo el $3 \%$ lo hace de manera excelente. Según la tabla de frecuencias, el punto (.) y la coma (,) son los signos que mejor saben usar. Por su parte, el punto y coma (;) es poco usado o mal empleado; y, presentan un nivel regular con respecto al uso de otros signos de puntuación (comillas, paréntesis, puntos suspensivos, raya, signos de admiración e interrogación, etc.).

Se evidencia que la mayoría de los estudiantes no hacen uso adecuado de los signos de puntuación cuando escriben, es decir, no estructuran el texto de manera que se pueda ver, como lo dicen Cassany et., al. (2003), el esqueleto jerárquico del texto ya que los apartados de este no quedan delimitados y se dificulta su comprensión. El punto y aparte es el signo mejor utilizado ya que se pueden observar los distintos párrafos en los textos.

En general, los resultados indican que los estudiantes presentan un nivel regular y bueno en el uso de los mecanismos de cohesión y que, por tanto, el conocimiento y aplicación de estos les permite estructurar textos en un nivel microestructural. Así las cosas, aunque no alcanzan un alto grado de conocimiento sobre la manera de estructurar los textos a nivel local, conocen la técnica y los procedimientos de construcción del texto, ensamblando las oraciones con distintos procedimientos léxicos, semánticos y gramaticales que dotan de sentido esas relaciones textuales (Sánchez, 2000).

\section{Coherencia}

Tabla 5

Frecuencia de uso de mecanismos de coherencia

\begin{tabular}{|c|c|c|c|c|c|}
\hline \multirow{2}{*}{ INDICADOR } & \multicolumn{4}{|c|}{ CALIFICACIÓN } & \multirow{2}{*}{ TOTAL } \\
\hline & $\mathbf{M}$ & $\mathbf{R}$ & B & $\mathbf{E}$ & \\
\hline Las ideas son claras y comprensibles. & 6 & 30 & 12 & 2 & 50 \\
\hline $\begin{array}{l}\text { Hay relación semántica adecuada entre las proposiciones } \\
\text { de la secuencia discursiva. }\end{array}$ & 6 & 27 & 15 & 2 & 50 \\
\hline $\begin{array}{l}\text { Las ideas se exponen de forma completa, progresiva y } \\
\text { ordenada. }\end{array}$ & 9 & 25 & 14 & 2 & 50 \\
\hline $\begin{array}{l}\text { Cada idea se desarrolla en un párrafo o unidad } \\
\text { independiente. }\end{array}$ & 12 & 26 & 10 & 2 & 50 \\
\hline $\begin{array}{l}\text { La información que expone en las partes del texto es } \\
\text { pertinente para el tema global. }\end{array}$ & 10 & 25 & 12 & 3 & 50 \\
\hline $\begin{array}{l}\text { Introduce citas en el texto y las parafrasea o explica de } \\
\text { manera precisa. }\end{array}$ & 22 & 20 & 8 & 0 & 50 \\
\hline Porcentaje & $22 \%$ & $51 \%$ & $24 \%$ & $3 \%$ & $100 \%$ \\
\hline
\end{tabular}

Fuente: elaboración propia (2021)

Nota: La tabla 5 indica que el $51 \%$ de los estudiantes escribe textos cuyo nivel de coherencia es regular, el $24 \%$ tiene un nivel bueno, el $22 \%$ malo y solo el $3 \%$ tiene un nivel excelente.

Lo anterior demuestra que, al momento de producir textos, los estudiantes presentan un 
nivel regular en el uso de los mecanismos de coherencia, es decir, las ideas que desarrollan no son comprensibles totalmente (se tornan confusas); también presentan dificultades para relacionar dichas ideas de forma global y así otorgarle unidad semántica al texto, lo que se debe, en gran medida, a que no las ordenan adecuadamente y no las exponen de forma completa y progresiva.

Por otro lado, presentan dificultades para desarrollar las ideas en unidades independientes, es decir, en varios párrafos, ya que suelen, en un mismo párrafo, exponer ideas que, aunque relacionadas, no guardan una continuidad de sentido con respecto a la idea principal del mismo. Además, las ideas que exponen en las partes del texto no son del todo pertinentes para el tema y contenido global de este, ya que dan información que no es necesaria para el asunto tratado, ocasionando una sobreproducción de ideas que, o bien redundan, o bien hacen perder la unidad semántica del texto.

Así pues, si se tiene en cuenta lo que sugiere Cassany (2003, p. 319), que "la coherencia establece cuál es la información pertinente que se ha de comunicar y cómo se ha de hacer (en qué orden, con qué grado de precisión o detalle, con qué estructura, etc.)" se puede aseverar que los estudiantes no tienen un conocimiento claro acerca de los mecanismos o formas de dar coherencia a los textos que producen, esto es, una coherencia global, que, según Van Dijk (1996) caracteriza un texto como un todo, y no como una simple suma de oraciones, o sea, una suma de partes no congruentes.

En conclusión, la falta de un amplio conocimiento y, por tanto, de ejecución de las formas de hacer coherente un escrito, constituyen uno de los mayores problemas de estos estudiantes para la producción de textos que sean comprensibles para los lectores.

\section{Superestructura}

Tabla 6.

Frecuencia de organización superestructural

\begin{tabular}{|c|c|c|c|c|c|}
\hline \multirow{2}{*}{ INDICADOR } & \multicolumn{4}{|c|}{ CALIFICACIÓN } & \multirow{2}{*}{ TOTAL } \\
\hline & $\mathbf{M}$ & $\mathbf{R}$ & B & $\mathbf{E}$ & \\
\hline $\begin{array}{l}\text { Jerarquiza y organiza las ideas del texto de } \\
\text { acuerdo con la tipología del mismo. }\end{array}$ & 19 & 21 & 7 & 3 & 50 \\
\hline $\begin{array}{l}\text { Cada parte de la superestructura cumple con } \\
\text { su función. }\end{array}$ & 22 & 19 & 3 & 6 & 50 \\
\hline Porcentaje & $41 \%$ & $40 \%$ & $10 \%$ & $9 \%$ & $100 \%$ \\
\hline
\end{tabular}

Fuente: elaboración propia (2021)

Nota: Como se aprecia, el $41 \%$ de los estudiantes estructura mal sus textos de acuerdo con la tipología del mismo, el $40 \%$ lo hace de manera regular, el $10 \%$ lo hace bien y, solo el $9 \%$, lo hace de manera excelente. 
Los datos demuestran que la mayor parte de los estudiantes presentan dificultades para construir sus textos en un nivel superestructural. Esto indica que no jerarquizan u organizan la información según la tipología textual y, por tanto, cada parte de la superestructura no cumple a cabalidad con su función.

Para el caso del ensayo, esto se traduce en el hecho de que no plantean la tesis y cuando tratan de hacerlo no se puede notar, con certeza, el punto de vista que pretenden defender. Al respecto, Agudelo (2001, p. 1) plantea que "la superestructura del ensayo quedaría mutilada sin la presencia de la tesis y aunque no hay impedimento para que ella sea tácita, sí es recomendable que se la presente directamente y de manera sencilla y precisa".

De igual forma, no hay un desarrollo argumental suficiente, progresivo y acertado. En algunas ocasiones introducen citas, las cuales no utilizan para construir argumentos de autoridad, o lo hacen inadecuadamente, es decir, que, en vez de soportar la tesis con razones y argumentos válidos, lo que hacen es generar confusión y falta de convicción en el lector.

En cuanto a la conclusión, se puede notar que, en la mayoría de los casos, se presenta un argumento más o una información nueva que no retoma la tesis o reflexiona acerca de esta o del cuerpo argumental desarrollado anteriormente en el texto.

En definitiva, la superestructura de los ensayos no estaba del todo acorde con las exigencias de ese tipo de texto debido a que la tesis no estaba bien planteada, por lo que es lógico que los argumentos fueran pobres o no tuvieran una coherencia total. Esto conlleva a que el lector no tenga claro de qué se trata el texto y cuál es su intención.

\section{Adecuación}

Tabla 7.

Frecuencia de mecanismos de adecuación

\begin{tabular}{lccccc}
\multicolumn{1}{c}{ INDICADOR } & \multicolumn{3}{c}{ CALIFICACIÓN } & TOTAL \\
\cline { 2 - 4 } & $\mathbf{M}$ & $\mathbf{R}$ & $\mathbf{B}$ & $\mathbf{E}$ & \\
\hline $\begin{array}{l}\text { El texto consigue el propósito } \\
\begin{array}{l}\text { comunicativo para el cual ha sido } \\
\text { producido. }\end{array}\end{array}$ & 8 & 30 & 10 & 2 & 50 \\
$\begin{array}{l}\text { Se mantiene un mismo nivel de lenguaje } \\
\text { durante todo el texto. }\end{array}$ & 5 & 19 & 21 & 5 & 50 \\
$\begin{array}{l}\text { El texto responde a la situación de } \\
\text { enunciación en la que fue producido. }\end{array}$ & 8 & 22 & 15 & 5 & 50 \\
\hline \multicolumn{1}{c}{ Porcentaje } & $14 \%$ & $47 \%$ & $30 \%$ & $9 \%$ & $100 \%$
\end{tabular}

Fuente: elaboración propia (2021)

Nota: La tabla 7 muestra que el $47 \%$ de los estudiantes presenta un nivel regular en cuanto a la adecuación de los textos de acuerdo con su contexto de producción, el 30\% lo hace bien, el $14 \%$ mal y solo el $9 \%$ lo hace de manera excelente.

los textos que produce, de acuerdo con la situación comunicativa; sin embargo, se 
esperaría que lo hicieran de manera excelente para alcanzar el propósito comunicativo propuesto, teniendo en cuenta que todo texto se circunscribe en un contexto pragmático y no puede ser ajeno a él. En el caso del ensayo, cuyo objetivo es defender una tesis para convencer o persuadir a una audiencia, la falencia se debe a que presentan dificultades para construir dicha tesis, por tanto, no queda claro el propósito comunicativo del texto.

Con respecto al nivel de lenguaje que se presenta en el texto analizado, se puede notar que una gran parte de los estudiantes tienen un nivel bueno (21) y un nivel regular (19). Los primero mantienen el nivel del lenguaje adecuado a la situación de comunicación, mientras que el segundo grupo mostró un tratamiento del lenguaje informal y poco técnico. De acuerdo con el manejo de los conocimientos específicos de las asignaturas que cursaban, se esperaba que dieran cuenta de un lenguaje más técnico al momento de referirse a los conceptos o al tema planteado.

Así pues, tal como lo plantean Cassany et., al. (2003), si se está escribiendo acerca de un tema especializado, lo más conveniente es escribir en la terminología específica de ese campo y evitar expresiones que sean coloquiales o muy generales ya que se pierde la precisión.

Asimismo, en los ensayos analizados no era común ver una situación de enunciación concreta en la que, a consideración de Martínez et., al. (2004), se evidenciara una configuración discursiva que construyera relaciones de sentido y voces enunciativas o puntos de vista. Así, por ejemplo, en algunos casos no era notable la presencia de puntos de vista en el discurso acerca del tema tratado, tanto propios como ajenos, por lo que no era clara la construcción de un enunciatario.

Estas dificultades en la adecuación textual están dadas, en mayor medida, por la falta de construcción de un texto coherente, con una superestructura bien definida y una situación de enunciación concreta. Vásquez (2016) señala, en términos muy sencillos, lo que sucede con los estudiantes cuando se enfrentan a la tarea de escribir ensayos:

Sin embargo, lo que se evidencia al recibir las tareas ensayísticas es su bajo nivel argumentativo, además de la falta de coherencia entre los diversos apartados del texto. Son escritos desvertebrados, sin ilación entre las ideas y sin ninguna tesis de base que invite a tomar partido o a confirmar un desacuerdo (p. 17)

En este punto, es necesario tener en cuenta, para la adecuación de un texto, las fases del modelo procesual de escritura de Flower y Hayes (1992), sobre todo la fase de planeación, para que antes de comenzar a escribir, se defina el propósito comunicativo, se pueda escoger el tipo de texto y de lenguaje más adecuado con ese fin, lo que permite a su vez construir una situación de enunciación coherente con ese propósito.

Para concluir con este estudio, se puede afirmar que lo adecuado sería que los estudiantes tuvieran un excelente 0 , al menos, un buen nivel en cada uno de los indicadores 
formulados para evidenciar su alto grado de conocimiento y práctica de los mecanismos de cohesión, coherencia y adecuación para la producción de textos académicos como producto de lo aprendido en la asignatura de Competencias Comunicativas.

\section{Discusiones y conclusiones}

El trabajo de investigación realizado evidencia, groso modo, que los estudiantes tienen un conocimiento amplio de los mecanismos de cohesión textual utilizados para referenciar elementos a nivel local, así como una buena práctica de uso de los mismos; sin embargo, cuando se trata de articular el texto como un todo coherente no formulan sus ideas de modo que se mantenga la unidad textual con una macroestructura concreta. Este problema dificulta la configuración enunciativa del texto, pues las ideas expuestas no siempre expresan, de manera precisa, los puntos de vista que el autor propone. En definitiva, no cumplen plenamente los requisitos que todo texto debe plasmar en tanto que manifestación lingüística humana para ser comprendido por sus interlocutores en su contexto específico de producción (Serafini, 1994; Van Dijk, 1996; Sánchez, 2000; Martínez, 2009; Cassany et. al., 2003; García 2015).

Dado lo anterior, se pone de manifiesto la falta de práctica de la escritura como proceso (Flower y Hayes, 1992), enfoque que resulta pertinente ya que se ha demostrado su efectividad para fortalecer la escritura de textos, haciéndolos más coherentes y adecuados (Briceño, 2014; Medina, Leal, Flórez y Rojas, 2009; Moreno, 2020). De tal manera, que textos incoherentes, no adecuados y no estructurados de acuerdo con las exigencias de una situación comunicativa, demuestran una falta de planificación, traducción, revisión y control constante que permita una escritura autorregulada.

Por lo tanto, es necesario que se realicen ajustes al programa de la asignatura de Competencias Comunicativas, en los que se tengan en cuenta los resultados de esta investigación para mejorar la competencia de escritura de los estudiantes, de modo que ello incida favorablemente en su formación y futuro ejercicio profesional.

Se propone que al iniciar el curso de competencias comunicativas, se realice una evaluación diagnóstica y se caractericen las prácticas de escritura que poseen los estudiantes, a través de encuestas, entrevistas, grupos focales u otras técnicas, para conocer el estado de esas competencias en los estudiantes y la manera como las desarrollan. Este acopio de información permitirá a los docentes seleccionar los contenidos y las actividades metodológicas más adecuadas para que el proceso de aprendizaje sea realmente significativo.

Así las cosas, los programas de competencias comunicativas, encargados de desarrollar este tipo de habilidades de escritura, deben diseñarse con base en teorías y modelos lingüísticos y didácticos actualizados, tales como los modelos discursivos (Martínez, 
et. al. 2004), modelos procesuales de escritura (Flower y Hayes, 1996; Bereiter y Scardamalia, 1992) y modelos didácticos con base en la pedagogía de géneros textuales (Álvarez, Benítez y Rosado, 2018) que le permitan al estudiante hacer una correcta construcción de significados y sentidos.

Es importante señalar que en el desarrollo de la competencia escritora hay una intersección de factores tanto pedagógicos y lingüísticos, como institucionales, que intervienen en la enseñanza y el aprendizaje de la escritura en el contexto universitario. Por ello, se deben considerar estos factores para la planificación, ejecución y evaluación de los programas de competencias comunicativas.

Ahora bien, con base en el análisis de los resultados se podrían plantear algunas líneas de reflexión adicionales basadas en cuestionamiento como: ¿cuáles son los lineamientos institucionales relacionados con el desarrollo de competencias comunicativas?, ¿cuáles son los fundamentos epistemológicos del curso?, ¿qué metodología emplea el docente para enseñar el proceso de producción textual?, ¿cómo son evaluadas estas competencias en la asignatura? Las respuestas a estos interrogantes bien podrían merecer subsiguientes investigaciones, con base en las cuales los docentes investigadores del contexto universitario reflexionen en torno al proceso didáctico que se sigue para alcanzar los objetivos de la asignatura estudiada.

Finalmente, esta pesquisa convoca a seguir trabajando en una ruta poco explorada en el ámbito de la pedagogía de la escritura en el contexto universitario: la evaluación de los programas de asignaturas de competencias comunicativas. Y no solo desde la competencia misma de los estudiantes, sino desde la metodología del docente, las perspectivas teóricas asumidas y las necesidades de los estudiantes.

\section{Referencias}

Agudelo, C. (2001). ¿Cómo se hace un ensayo? (U. d. Caldas, Ed.). Hacia la promoción de la salud, (6), 1-3.

Álvarez, S., Benítez, T., \& Rosado, N. (2018). Español académico para aprender en las disciplinas. Implementación de un programa institucional para el desarrollo de la competencia comunicativa en la educación superior. Revista Nebrija de Lingüística Aplicada a la Enseñanza de Lenguas, 12(24), 32-55.

Andrade, M. (2009). La escritura y los universitarios. Universitas Humanística(68), 297-340.

Ávila, M., González, P., \& Peñaloza, C. (2013). Creación de un programa de escritura en una universidad chilena: estrategias para promover un cambio institucional. Revista Mexicana de Investigación Educativa, XVIII(57), 537-560. 
Bereiter, C., \& Scardamalia, M. (1992). Dos modelos explicativos de los procesos de composición escrita. Journal for the Study of Education and Development, Infancia $y$ Aprendizaje(58), 43-64.

Briceño, J. (2014). El modelo de Flower y Hayes: una estrategia para la enseñanza de la escritura académica. Universidad del Tolima.

Briones, G. (1996). Metodología de la investigación cuantitativa en las ciencias sociales. ICFES.

Cassany, D., Luna, M., \& Sanz, G. (2003). Enseñar Lengua (Novena ed.). Editorial Graó.

Colciencias. (2013). Para qué se lee y se escribe en la universidad colombiana. Un aporte a la consolidación de la cultura académica del país. Pontificia Universidad Javeriana.

Díaz, S., \& Morales, I. (2013). Intervención sociometacognitiva: una apuesta para la comprensión textual. Revista Iberoamericana de Educación, 61(4), 1-13.

Eudaye, D., Carvajal, M., Macías, A., Páez, D., \& Cañedo, T. (2019). Sistematización de experiencias de prácticas de escritura en tres programas de pregrado. Investigación cualitativa en educación, (1), 991-1000.

Fernández, A. (2019). Educación: competencias, emociones y humor, perspectivas y estudios. Educación y Humanismo, 21(37), 51-66. https://doi.org/10.17081/eduhum.21.37.3365

Flores, M. (2018). La escritura académica en estudios de ingeniería: valoraciones de estudiantes y profesores. Revista de la Educación Superior, 47(186),23-49. https://doi.org/10.36857/resu.2018.186.344

Flower, L., \& Hayes, J. (1996). La teoría de la redacción como proceso cognitivo. Textos en contexto. Los procesos de lectura y escritura, (1),3-19.

García, F. (2015). Comprensión lectora y Producción textual. Ediciones de la U.

González, B., \& Vega, V. (2010). Prácticas de lectura y escritura en cinco asignaturas de diferentes programas de la Universidad Sergio Arboleda. Civilizar. Ciencias Sociales y Humanas, XX(18), 101-116. http://www.redalyc.org/articulo.oa?id=100220339009

Hymes, D. (1996). Acerca de la Competencia Comunicativa. Forma y Función, (9), 13-37.

Londoño, D., \& Ospina, M. (2018). Comprensión y producción de textos escritos en instituciones de educación superior. Trilogía, Ciencia, Tecnología, Sociedad, 10(18), 183-202. https://doi.org/10.22430/21457778.671

Martínez, M., Álvarez, D., Hernández, F., Zapata, F., \& Castillo, L. (2004). Discurso y Aprendizaje. Universidad del Valle. 
Medina, M., Leal, A., Flórez, R., \& Rojas, S. (2009). Efectos de un programa basado en los postulados de la lectura y la escritura como proceso sobre la calidad de comprensión y producción de textos expositivos en niños de tercero y cuarto de primaria. En $A$. Velasco, E. Mondragón, H. Suárez, \& M. Osorio, La lectura y la escritura como procesos transversales en la escuela (pp. 31-62). Instituto para la Investigación Educativa y el Desarrollo Pedagógico, IDEP.

Ministerio de Educación Nacional. (1998). Lineamientos curriculares de Lengua Castellana. Ministerio de Educación Nacional.

Ministerio de Educación Nacional. (2006). Estándares Básicos de Competencias en Lenguaje, Matemáticas, Ciencias y Ciudadanas. Ministerio de Educación Nacional.

Molina, V., \& López, K. (2020). Estado de la cuestión de los centros y programas de escritura de Latinoamérica. Revista Colombiana de Educación, 1(78), 97-119.

Moreno, J. (2020). La composición escritural expositiva desde el modleo psicolingüístico de Flower y Hayes: Estrategias para su fortalecimiento. Quaestiones Disputatae: Temas En Debate, 13(27), 55-79.

Moyano, E. (2016). Un programa de lectura y escritura universitario: lineamientos teóricos, características y resultados de aplicación. Grafía, 13(1),33-59.

Moyano, E. (2018). Diseño e implementación de programas de lectura y escritura en el nivel universitario: principios y estrategias. Lenguas Modernas, (50), 47-72. https://lenguasmodernas.uchile.cl/index.php/LM/article/view/49251/51717

Pérez, H. (2006). Comprensión y Producción de Textos Educativos. Editorial Magisterio.

Ramírez, M., Tánori, J., \& García, R. (2017). Evaluación de la escritura argumentativa de estudiantes universitarios del área de educación. Educación Superior , (23), 77-88.

Rivera, M., Cornejo, C., \& Roco, A. (2008). Evaluación de la competencia lectora en estudiantes de primer año de carreras del área humanista y carreras del área de la salud en tres universidades del Consejo de Rectores. Estudios Pedagógicos, XXXIV(1), 123-138.

Rojas, G., \& Rodríguez, E. (2018). Creencias y prácticas de escritura. Comparación entre distintas comunidades académicas. Revista Mexicana de Investigación Educativa, 23(79), 1093-1119.

Sánchez, A. (2000). Redacción Avanzada. International Thomson Editores.

Serafini, M. (2011). Cómo se escribe. Paidós.

Suárez, T. (2019). Estudio explicativo sobre el efecto de un programa para el desarrollo de 
competencias argumentativas orales y escritas en estudiantes universitarios de la C.U.I. Corporación Universitaria Iberoamericana.

Van Dijk, T. (1992). La ciencia del texto (Tercera ed.). Ediciones Paidós.

Van Dijk, T. (1996). Estructuras y Funciones del Discurso. Siglo XXI Editores.

Vásquez, F. (2016). Las claves del ensayo. Editorial Kimpress. 